\title{
agitation after general anesthesia in children
}

Saeko Miyazaki, Takahiro Mihara, Maai Hino, Takahisa Goto, Koui Ka

Department of Anesthesiology, Kanagawa Children's Medical Center

\section{Background}

Emergence agitation (EA) is a common complication after general anesthesia in children.

The aim of this study was to develop an EA risk score by two-phased (development and validation phases) study.

\section{Methods}

\section{Development phase}

Analyzed the data from our past $\mathrm{RCT}^{*}$ by using logistic regression.

Determined the best predictive model for EA.

\section{Validation phase}

Assessed the incidence of EA using a PAED scale.

$>$ Examined the predictive ability of the EA risk score by generating a receiver operating characteristic (ROC) curve.

\section{Results}

Subjects (common to both phases)

Patients: children aged $1.5-9$ years

Operations: minor surgeries**

Anaesthesia: general anaesthesia with sevoflurane (and regional anaesthesia)

*The effect of meridian stimulation on EA after sevoflurane anaesthesia was studied with 120 children. (Eur J Anaesthesiol 2015)

**inguinal hernia repair, umbilical hernia repair, cryptorchidism repair, scrotum edema, adenoidectomy, tonsillectomy, tubing, and strabismus surgery

A) Characteristic

\begin{tabular}{lll}
\hline & Phase1 $(\mathbf{n}=\mathbf{1 2 0})$ & Phase2 $(\mathbf{n}=\mathbf{8 1})$ \\
\hline age (months) & $48.5(33.8-64.5)$ & $54.5(33-72)$ \\
sex (male:female) & $88: 37$ & $55: 26$ \\
weight (kg) & $15.8(13.2-18.4)$ & $15.4(12.6-19)$ \\
Type of operation & & \\
$\quad$ Strabismus surgery & 12 & 5 \\
$\quad$ Tonsillectomy & 16 & 12 \\
$\quad$ Inguinal hernia repair & 33 & 46 \\
$\quad$ Others & 59 & 18 \\
Anaesthesia time (min) & $76.5(47-99)$ & $60(42-92)$ \\
\hline
\end{tabular}

\section{B) The EA risk score}

\begin{tabular}{|c|c|}
\hline & Score \\
\hline Age (year) & 9-Age \\
\hline \multicolumn{2}{|l|}{ Operative procedure } \\
\hline Strabismus surgery & 7 \\
\hline Tonsillectomy & 7 \\
\hline Others & 0 \\
\hline \multicolumn{2}{|l|}{ Preoperative behavior score } \\
\hline Screaming or shouting & 4 \\
\hline $\begin{array}{l}\text { Tearful and/or withdrawn } \\
\text { but compliant with induction }\end{array}$ & 2 \\
\hline Calm and controlled & 0 \\
\hline \multicolumn{2}{|l|}{ Anaesthesia time } \\
\hline$>2$ hours & 4 \\
\hline 1 to 2 hours & 2 \\
\hline$<1$ hour & 0 \\
\hline The EA & k score \\
\hline
\end{tabular}

The score ranged 0-23 points and included four factors.

\section{C) The ROC curve}

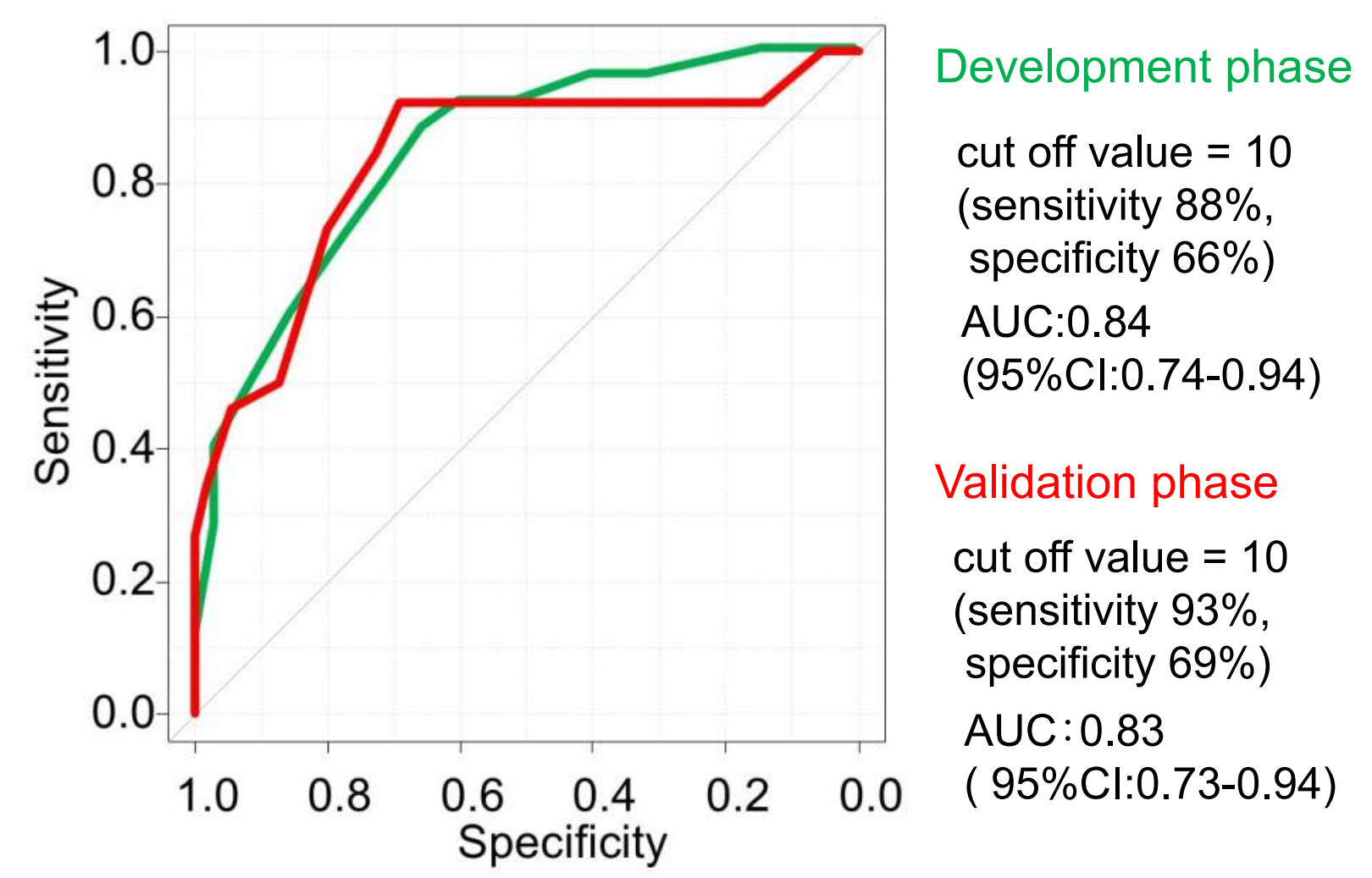

$>$ The similar results between the different set of patients indicated the validity of the EA risk score. 